\title{
Microstereolithography of Three-Dimensional Polymeric Springs for Vibration Energy Harvesting
}

\author{
Evan Baker, Timothy Reissman, Fan Zhou, Chen Wang, Kevin Lynch, and Cheng Sun \\ Department of Mechanical Engineering, Northwestern University, Evanston, IL 60208-3111, USA \\ Correspondence should be addressed to Cheng Sun, c-sun@northwestern.edu \\ Received 17 December 2011; Accepted 13 March 2012 \\ Academic Editor: Einar Halvorsen \\ Copyright () 2012 Evan Baker et al. This is an open access article distributed under the Creative Commons Attribution License, \\ which permits unrestricted use, distribution, and reproduction in any medium, provided the original work is properly cited.
}

The inefficiency in converting low frequency vibration $(6 \sim 240 \mathrm{~Hz})$ to electrical energy remains a key issue for miniaturized vibration energy harvesting devices. To address this subject, this paper reports on the novel, three-dimensional micro-fabrication of spring elements within such devices, in order to achieve resonances and maximum energy conversion within these common frequencies. The process, known as projection microstereolithography, is exploited to fabricate polymer-based springs direct from computer-aided designs using digital masks and ultraviolet-curable resins. Using this process, a micro-spring structure is fabricated consisting of a two-by-two array of three-dimensional, constant-pitch helical coils made from 1,6-hexanediol diacrylate. Integrating the spring structure into an electromagnetic device, with a magnetic load mass of 1.236 grams, the resonance is measured at $61 \mathrm{~Hz}$, which is within $2 \%$ of the theoretical model. The device provides a maximum normalized power output of $9.14 \mu \mathrm{W} / \mathrm{G}\left(G=9.81 \mathrm{~ms}^{-2}\right)$ and an open circuit normalized voltage output of $621 \mathrm{mV} / \mathrm{G}$. To the best of the authors knowledge, notable features of this work include the lowest Young's modulus $(530 \mathrm{MPa})$, density $\left(1.011 \mathrm{~g} / \mathrm{cm}^{3}\right)$, and "largest feature size" $(3.4 \mathrm{~mm})$ for a spring element in a vibration energy harvesting device with sub-100 Hz resonance.

\section{Introduction}

The need for miniaturized, multifunctional electromechanical structures and material systems with the capability to harvest energy from the environment has grown significantly in recent years in response to the proliferation of portable electronic devices and wireless sensors [1-4]. At the present time, batteries are the primary power source for these portable devices. Unfortunately, batteries suffer from a limited lifetime requiring periodic recharging or replacement [5]. Thus, harvesting energy from the environment offers an autonomous means to recharge or directly power conventionally battery-operated devices. In evaluating the available energy harvesting technologies, solar cells are the most mature. However, their dependence on sunlight restricts the locations where solar cells can be effective. In contrast, environmental vibration is a particularly attractive energy source because of its abundance in nearly all environments, spanning a wide frequency range [6]. This has led to the research field known as vibration energy harvesting (VEH), which explores using different principles of transduction to convert available vibration energy into electricity [7-11].

In attempting to miniaturize VEH devices, a major issue has arisen with the mismatch between device resonant frequencies and the environmental vibration frequency spectrum. The available energy range for most VEH applications is between $6 \mathrm{~Hz}$ and $240 \mathrm{~Hz}$ [6]. However, current microfabrication techniques used have been limited to primarily two-dimensional (2D) topologies and to using materials with high Young's moduli, such as piezoceramics and silicon, for their spring components. Consequently, these devices have high resonant frequencies and can optimally harvest energy only at the kilohertz frequency range and above $[5,12]$. Thus, reducing the operation frequency range to the available energy range requires proportionally scaling up the device dimensions or adding an excessive amount of mass [6]. These options run counter to the motivation of using this technology as miniaturized power sources.

To understand the fundamental scaling issue, let us consider the simple model of a linear, undamped spring-mass 
system with a spring $k$, a mass $m$, and a base-excitation force $F(\omega)$, see Figure 1. In solving for the relative displacement $x$ of the mass, we find that $x(\omega)$ is infinite at resonance, $\omega=\sqrt{k / m}$. Thus, to achieve optimal performance of a VEH device, which has an assumed small damping present, requires precise tuning of the ratio of the spring constant $k$ and mass $m$ to be near resonance. With traditional silicon micromachining processes, geometries are limited to suspended 2D structures, which results in many designs fundamentally reducing to cantilever beam configurations in order to achieve low flexural rigidity. With such a configuration, the spring constants are proportional to a function of the maximum feature length, resulting in low resonance designs which are small in volume but large in a single direction. Additionally, for many engineering materials used in traditional microfabrication, the properties of Young's modulus and density are correlated along the diagonal line, as evidenced by the well-known Ashby chart.

Thus, reducing the $\mathrm{k} / \mathrm{m}$ ratio requires an external mass which is proportional to the VEH's Young's modulus. If that Young's modulus is high, a significantly large mass will be needed to lower the resonance, resulting in larger system dimensions. Therefore, to address the seeming contradictory requirement of low resonant frequency and device miniaturization, projection microstereolithography ( $\mathrm{P} \mu \mathrm{SL})[13]$ is introduced in this paper as a means to widen the design space via three-dimensional (3D) microfabrication technology and to lower the Young's modulus away from the bulk (rigid) and towards the green (compliant) state [14], requiring less mass to tune the resonance.

Within this work, the detailed design of a 3D spring structure for a microelectromagnetic VEH is given. The selection of an electromagnetic design was made since this conversion has been shown to be comparable at the small scale to the widely used piezoelectric transduction [15]. To begin, the spring-mass structure is designed to match the resonance of a specific environmental application, the ambient vibration of devices operating at $60 \mathrm{~Hz}$, the designated US electrical line frequency. Using $\mathrm{P} \mu \mathrm{SL}$, the design space is expanded to $3 \mathrm{D}$, and manipulation of the photopolymer spring material is used to tailor the Young's moduli within the range from green- to bulk-state properties [14]. In this work, the model spring-mass structure fabricated and tested is an array of four constant-pitch helical coils, with a maximum spring feature size of only $3.4 \mathrm{~mm}$ and an external magnetic load of 1.236 grams. Using this approach, miniaturization of an electromagnetic $\mathrm{VEH}$ with low resonance frequency and small feature size is demonstrated. Additionally, it is noted that implementation of $\mathrm{P} \mu \mathrm{SL}$ is not limited to just electromagnetic devices, but rather can be applied as spring components in other transduction types such as electrostatic or piezoelectric, making it an option for miniaturization of all VEH.

The paper is organized as follows. The P $\mu \mathrm{SL}$ fabrication process is detailed in Section 2. In Section 3, analytical theory on the voltage and power output of an electromagnetic VEH is given, which emphasizes the importance of matching the resonance of the structure with the vibration frequency of the source. Additionally, within this section the parameters

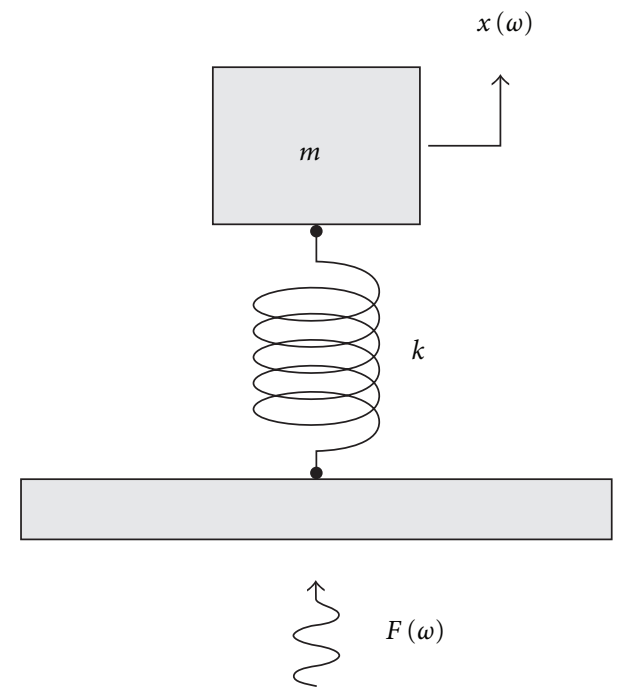

FIGURE 1: Schematic drawing of a simplified spring-mass system with a vibration source.

important to the $3 \mathrm{D}$ helical spring design are given. In Section 4, the experimental setup is introduced. In Section 5, experimental results are given as well as a comparison made to the theory of Section 3. In Section 6, we discuss the results with comparisons made to the literature.

\section{Projection Microstereolithography Process}

2.1. Principle. In recent years, the development of computeraided design (CAD) powered rapid prototyping, 3D printing, and direct writing techniques has made 3D fabrication in macroscale $(>1 \mathrm{~mm})$ possible $[13,16-18]$. Examples within PowerMEMS technology using P $\mathrm{SL}$ have been applied to sensing and actuating [19-21]. Here this technology is extended to generator applications, specifically VEH. To explain this process, microstereolithography ( $\mu \mathrm{SL})$ works by building microstructures from a photo-curable resin in a layer-by-layer fashion. The polymer layers are constructed by scanning a focused laser spot on the photo-curable resin surface. Despite its broader range of applications in microfluidic systems, integrated micro-optics, and biomedical engineering, its point-by-point scanning nature seriously constrains the process throughput, making it costly for practical device fabrication. To reduce fabrication time and cost, projection microstereolithography $(\mathrm{P} \mu \mathrm{SL})$ was developed to parallelize the $3 \mathrm{D}$ microfabrication process, see Figure 2. The core of this technology rests on the use of a spatial light modulator (SLM) as a dynamic mask. It can be either a liquid crystal display (LCD) panel or a digital micromirror device (DMD, Texas Instrument) panel. In contrast to fabricating $3 \mathrm{D}$ structures in a point-by-point scanning fashion, $\mathrm{P} \mu \mathrm{SL}$ uses a dynamic mask $(1400 \times 1050$ pixels $)$ to fabricate an entire $2 \mathrm{D}$ pattern in a single exposure. The electronic masks representing the constructed layers can be determined either by slicing a 3D CAD model into a series of closely spaced horizontal planes, or they can be generated by programming codes. 


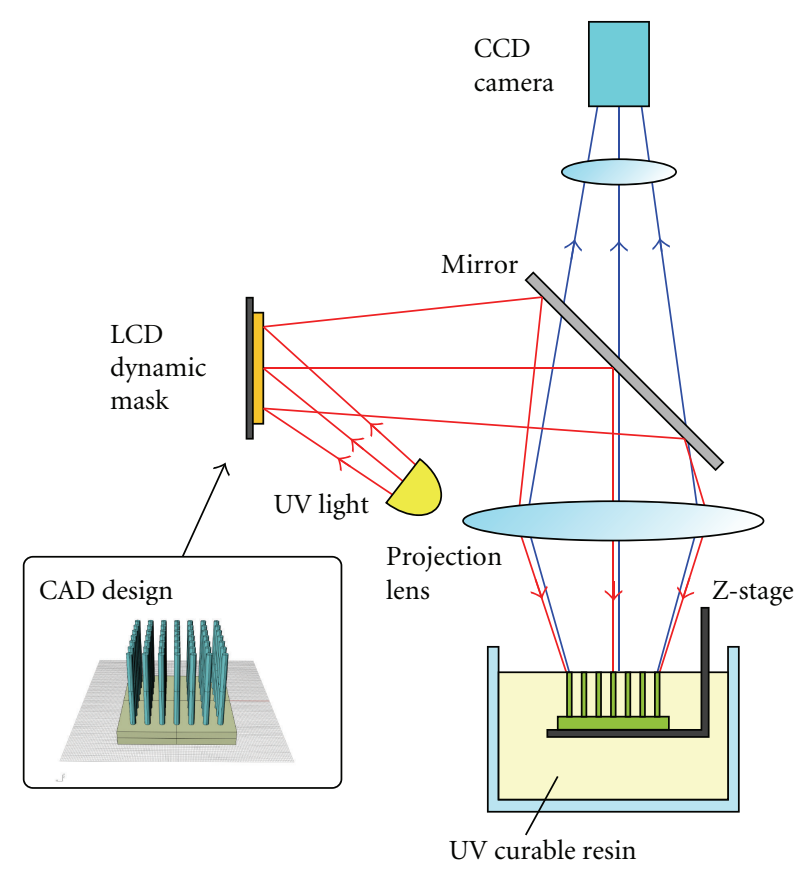

Figure 2: Schematic diagram of $\mathrm{P} \mu \mathrm{SL}$ system that uses a dynamic mask. A 3D CAD design is sliced into serials of 2D pattern and then displayed as a bitmap image on the dynamic mask. The demagnified image is projected on curable monomer resin surface to define the shape of solidified layers. By sequentially building 2D layers from bottom to the top, complex 3D microstructures are fabricated.

These dynamic mask patterns are dynamically displayed as bitmap images on the programmable dynamic mask, which is located at the uniformly illuminated plane of the ultraviolet (UV) light source to ensure a uniform light distribution on the image plane. Using a dynamic mask greatly simplifies the fabrication process, eliminating the need for mask fabrication. The UV light illuminated on the patterned mask is shaped, passes through a reduction lens, and finally projects on the top surface of photo-curable polymer resin in high resolution, with each pixel corresponding to $7.1 \times$ $7.1 \mu \mathrm{m}^{2}$. Meanwhile, the light reflected from the resin surface is collected by the projection lens and reflected by the beam splitter to the CCD camera, which is used to monitor the focusing of the image. The substrate is mounted on the $\mathrm{Z}$ stage, which can move in the vertical direction. Another $\mathrm{Z}$ stage is used to mount the container and adjust the height of the liquid surface. The liquid resin under the illuminated area is cured into a solid layer and glued onto the substrate. The mask is then switched to black to prevent further exposure, and the structure is lowered by a motorized $\mathrm{Z}$ stage to allow the next liquid layer to be covered on top. By repeating the fabrication step for each layer, highly complicated 3D structures can be accomplished from the bottom-up rapidly according to the designed geometry.

The system is compatible with various photopolymers. For the microfabrication in this paper, the UV curable resin used contains 1,6-hexanediol diacrylate (HDDA, Sigma-Aldrich) as the low-viscosity monomer and $2 \%$ photoinitiator (Irgacure 819, Ciba). Moreover, $0.22 \%$ UV absorber (Sudan I, Sigma-Aldrich) has been mixed with the UV curable resin to control the curing depth to be approximately $20 \mu \mathrm{m}$.

In 2001 Manias et al. [14] demonstrated the Young's modulus of a photopolymer could be tuned using the $\mu \mathrm{SL}$ technique over a broad range $(200-930 \mathrm{MPa})$ by adjusting the UV after cure time. The postcuring process occurs after the structure is removed from the nitrogen-enriched chamber and the liquid polymer resin within the structure has not completely solidified. It was found that by varying the immersion time of the structure under a UV lamp from zero to 360 minutes at an exposure rate of $5 \mathrm{Wm}^{-2}$, the rigidity of the material could be further manipulated. Thus, $\mathrm{P} \mu \mathrm{SL}$ combined with the postcuring process allows the Young's modulus to be tuned anywhere from the green- to bulk-state properties, offering even smaller, more compact structures with low resonances.

2.2. 3D Microfabrication of Spring Structures. Taking advantage of the 3D fabrication capability in $\mathrm{P} \mu \mathrm{SL}$ and the tunable Young's modulus, an array of polymeric helical coils was designed and fabricated to serve as a compliant spring structure for $\mathrm{VEH}$, in order to lower the resonance frequency to $60 \mathrm{~Hz}$ while maintaining the device compactness. The 3D CAD design of the spring structure is shown in Figure 3(a). To improve the load stability of the structure, the helical coils are arranged in a symmetric two-by-two form. Below the spring structures, the flat substrate is fabricated using a same process. At the top of springs, a grid-like platform is formed to support the magnetic load mass needed for the electromagnetic VEH design. The complete structure is made of 170 layers, with each layer being $20 \mu \mathrm{m}$ thick, yielding a total structure height of $3.4 \mathrm{~mm}$. The designed geometry of an individual helical coil is illustrated in Figure 3(b). The coil diameter $D$, width $b$, and thickness $t$ are $1040 \mu \mathrm{m}, 400 \mu \mathrm{m}$, and $160 \mu \mathrm{m}$, respectively. A scanning electron microscope (SEM) image of the fabricated coil array is shown in Figure 3(c). The dimensions of the fabricated coil are measured as $D=1010 \mu \mathrm{m}, b=372 \mu \mathrm{m}$, and $t=163.5 \mu \mathrm{m}$. The dimensions are in good agreement with the design parameters, demonstrating the accuracy in rapid fabrication of complex 3D geometry using the $\mathrm{P} \mu \mathrm{SL}$ technique.

\section{Vibration Energy Harvester (VEH)}

With the fabrication of polymeric 3D structures using $\mathrm{P} \mu \mathrm{SL}$ demonstrated in the previous section, this section focuses on the analytics specific to the electromagnetic VEH. Specifically, maximum power generation is analyzed in order to emphasize the importance of matching the resonant frequency of the device with the vibration frequency. Additionally, the equation for the spring constant $k$ of the constant pitch helical structure from Figure 3 is given along with its maximum loading in order to determine the tunable resonant frequency range of the 3D spring design.

3.1. Linear Electromagnetic Generator. For single-axis relative motion within electromagnetic devices, recall that 


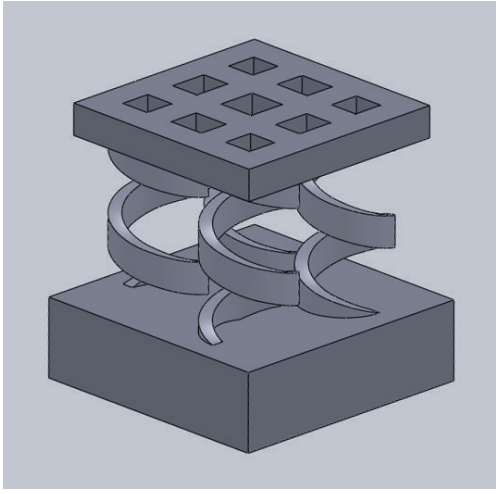

(a)

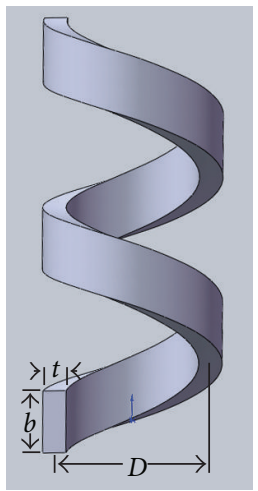

(b)

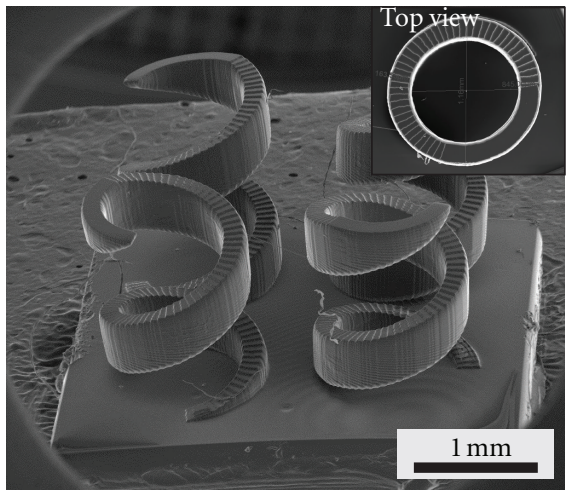

(c)

FIGURE 3: (a) A 3D CAD design of the polymeric soft spring structure. (b) The design geometry of an individual coil. (c) SEM image of coil array fabricated using the $\mathrm{P} \mu \mathrm{SL}$ method. Inset shows the top view of an individual coil.

the induced voltage can be calculated by applying the law of induction:

$$
V_{\text {ind }}=-\nabla \psi(\vec{x}) \cdot \frac{d \vec{x}}{d t}
$$

which states that the induced voltage $V_{\text {ind }}$ is a result of the time rate of change of the flux linkage of the magnetic coil $\psi$ with respect to the relative position $\vec{x}$ of the magnetic coil and the magnetic object. Since commonly the magnetic object is a dipole magnet, (1) is simplified by assuming harmonic motion, where $\omega$ is the vibration's angular frequency, and by substituting a coefficient in place of the flux gradient [22]:

$$
V_{\text {ind }}=\vec{K} \cdot \omega \vec{x} \text {. }
$$

Here, $\vec{K}$ is referred to as the electromagnetic coupling, which is equal to the cross-product of the magnetic coil's length $\vec{L}$ and the average magnetic flux density, $\vec{B}_{\text {ave }}$, applied to the length of the magnetic coil. Thus, the selection of the magnet with respect to its strength, along with the average of the magnetic coil's turns within the magnetic flux, determines the value for the electromagnetic coupling. To determine the power generation $P$, Ohm's law is used, and a voltage divider circuit is created with a resistive load $R_{l}$ applied to the voltage output of the magnetic coil. This yields

$$
P=\frac{R_{l}(\vec{K} \cdot \omega \vec{x})^{2}}{\left(R_{l}+R_{c}\right)^{2}},
$$

where $R_{c}$ is the magnetic coil's resistance. Thus, from a structural dynamics perspective, to maximize the voltage and power generation of an electromagnetic VEH at a fixed vibration frequency, the relative motion $\vec{x}$ of the magnetic coil and the magnetic object must be maximized. To predict the value for $\vec{x}$, the coupled system is modeled similar to Figure 1 but with a damper added to account for the parasitic damping and the electrical damping, which arises from the Lorentz force. Still assuming harmonic motion, the lumpedparameter model yields

$$
\vec{x}=\frac{\omega^{2} \vec{y}}{-\omega^{2}+\left(c_{p}+c_{e}\right) \omega / m+k / m},
$$

where $\vec{y}$ is the vibration, or base motion (assumed to be in the same direction as $\vec{x}), c_{p}$ is the parasitic damping constant, $c_{e}$ is the electrical damping constant, and $k$ is the spring constant. Here, we assume that the mass of the spring structure is negligible compared to the mass of the magnet, or moving object. From (4), it can be seen that to maximize $\vec{x}$, the system's undamped, natural angular frequency must match the vibration angular frequency, $\omega=\omega_{n}=\sqrt{\mathrm{k} / \mathrm{m}}$, which simplifies (4) to

$$
\vec{x}_{(\omega=\sqrt{k / m})}=\frac{m \omega \vec{y}}{\left(c_{p}+c_{e}\right)} .
$$

Combining (5) into (3), we observe the following relationship at resonance, assuming low damping:

$$
P_{(\omega=\sqrt{k / m})}=\frac{R_{l}}{\left(R_{l}+R_{c}\right)^{2}}|\vec{K}|^{2} \frac{m^{2} \omega^{4}|\vec{y}|^{2}}{\left(c_{p}+c_{e}\right)^{2}} .
$$

Here, it is noted that the damping constants and the electromechanical coupling are typically found experimentally. One simple method is to apply two impulse tests and record the logarithmic decrements. The first test is at the opencircuit condition (no electrical damping), which yields the parasitic damping coefficient $c_{p}$. The second test is with a known resistive load, which yields a different damping value due to the presence of the combined parasitic and electrical damping. Thus, comparing the two values, the value for $c_{e}$ can be calculated. With the value for $c_{e}$ known 


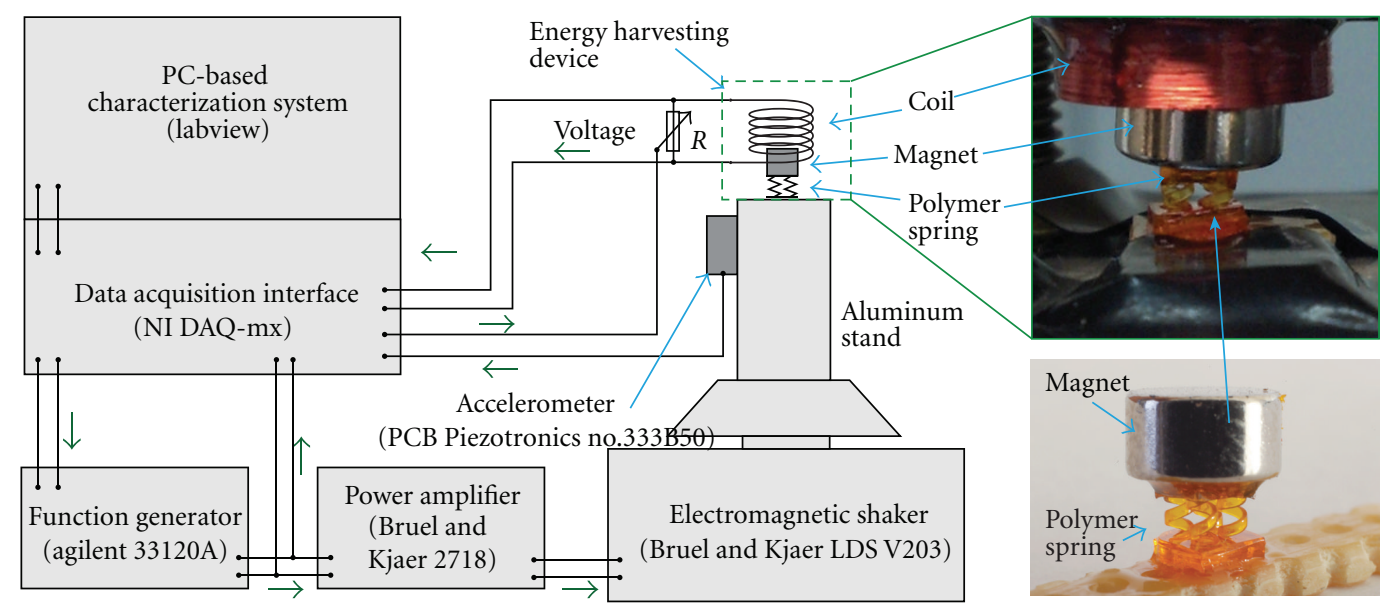

FIGURE 4: (left) experimental setup, (right) neodymium-ion-boron rare Earth Magnet(s) on top of the projection microstereoLithographyfabricated HDDA polymer $2 \times 2$ spring array structure (lower right, VEH with load mass, top right: with $1.236 \mathrm{~g}$ load mass and inside the 3000-turn 48 AWG magnet wire coil).

the electromechanical coupling $\vec{K}$ can then be determined as well [22]:

$$
|\vec{K}|^{2}=c_{e}\left(R_{l}+R_{c}\right)
$$

Thus, without knowing the damping constants and the electromechanical coupling a priori, the typical design approach for maximizing the voltage and power output is to tune the spring constant, as well as the acceptable mass loading, to yield a resonant structure at the vibration frequency.

3.2. PuSL Spring Design. As has been derived, it is important to match the resonant frequency of the device with the vibratory frequency of the application. Therefore for lowfrequency applications, a structural design with a low spring constant and the ability to hold a large load mass is ideal. For the former, the $\mathrm{P} \mu \mathrm{SL}$ process is used to expand the realm of geometric designs to $3 \mathrm{D}$ and to tailor the material properties via the length of the postprocess UV cure time [14]. For the latter, the maximum mass that the structure can hold under cyclical loading is determined based on the structural design and material properties chosen.

Using the helical spring design as the model structure in Figure 4 the analysis of the spring constant assumes that the response operates predominately in compression, which is reasonable given the typical sub-one $\mathrm{G}\left(1 \mathrm{G}=9.81 \mathrm{~ms}^{-2}\right)$ vibration forcing amplitudes observed in the literature [23]. Thus, for the design's rectangular cross-sectional geometry, the spring constant $k_{\text {helix }}$ for each helical coil within the structure is defined as

$$
k_{\text {helix }}=\frac{K_{2} E b t^{3}}{2(1+v) n D^{3}},
$$

where $K_{2}$ is the shape factor according to Table $1, E$ is the Young's modulus, $v$ is Poisson's ratio, $n$ is the number of spring turns, and $b, t, D$ remain the same as when they were defined in Section 2. The complete spring structure consists of four helical springs in parallel, which yields a structural spring constant $k=4 k_{\text {helix }}$. A CAD model displaying the helix geometry, as well as the complete structure, is shown in Figure 3.

With the structural spring constant known, the lower bound for the resonant frequency of the structure is determined based on the maximum mass $m_{\max }$ that can be applied under cyclical loading without exceeding its maximum stress $\sigma_{\max }$. To calculate this lower bound, the combined stress, which is the combination of the torsional and transverse shear stresses, is used, which is defined as

$$
m_{\max }=0.4 \frac{N \sigma_{\max } b t^{2}}{K_{w} K_{l} D \omega^{2} x},
$$

where $N$ represents the number of helical springs in parallel, $K_{w}$ is the Wahl factor as determined from Table 2, and $K_{l}$ is another shape factor according to Table 1 . The coefficient 0.4 is used when applied to cyclical loading cases. Thus, with the maximum stress of the material and the expected acceleration levels known, the range of acceptable tuning masses and their corresponding resonant frequencies can be calculated.

In conclusion, with $\mathrm{P} \mu \mathrm{SL}$ offering the ability to create $3 \mathrm{D}$ structures as well as manipulate the material properties, the spring constant of the helical spring structure can be predicted and tailored. The helical spring design represents just one of many $\mathrm{P} \mu \mathrm{SL}$ fabricated designs that can enable both compact and flexible spring structures with low resonant frequencies for vibration energy harvesting.

\section{Experimental Setup}

The experimental setup is designed to simulate environmental vibration sources, see Figure 4. To represent the general waveform of the vibration, a control signal is selected and generated by a function generator (Agilent 33120A). The control signal is then amplified by a power amplifier (Bruel and Kjaer no. 2718) before entering into an electromagnetic shaker (LDS V203). The resulting shaker's waveform and 
TABLE 1: Shape factor for rectangular cross-section.

\begin{tabular}{lccccccccccc}
\hline$b / t$ & 1.0 & 1.5 & 1.75 & 2.0 & 2.5 & 3.0 & 4.0 & 6.0 & 8.0 & 10.0 \\
\hline$K_{1}$ & 2.41 & 2.16 & 2.09 & 2.04 & 1.94 & 1.87 & 1.77 & 1.67 & 1.63 & 1.60 \\
$K_{2}$ & 0.18 & 0.25 & 0.272 & 0.292 & 0.317 & 0.335 & 0.385 & 0.381 & 0.391 & 0.399 \\
\hline
\end{tabular}

TABLE 2: Wahl factor.

\begin{tabular}{lccccccccccc}
\hline$D / t$ & 3 & 4 & 5 & 6 & 7 & 8 & 9 & 10 & 11 & 12 \\
\hline$K_{w}$ & 1.58 & 1.4 & 1.31 & 1.25 & 1.21 & 1.18 & 1.16 & 1.14 & 1.13 & 1.12 \\
\hline
\end{tabular}

acceleration are monitored by a LabVIEW program using a uniaxial accelerometer (PCB Piezotronics no. 333B50) mounted to the shaker drive output. An aluminum stand is used to position the device under testing (DUT) sufficiently away from the magnetic field of the shaker, so as to not to cause magnetic interference.

The base of the DUT is fixed to the top of the aluminum stand using adhesive. The device itself consists of three main components: the spring structure, a 1.236-gram magnetic load, and a 3000-turn coil (48 AWG magnet wire) with a resistance of $2.112 \mathrm{k} \Omega$. The magnetic load is comprised of three layers: a 0.5-gram tungsten mass sandwiched between two magnetically opposing 0.368 -gram $\mathrm{NdFeB}$ rare earth magnets. Each of the layers are fixed in place with epoxy and then attached to the top of the spring structure using epoxy again. The magnetic coil is positioned directly above the magnet such that the bottom of the coil is aligned with the top of the magnet, as seen in Figure 4. Lastly, the voltage output of the device is measured across a resistive load and recorded with LabVIEW.

Two different sets of vibration tests are performed to extract the electrical performance of the device as well as its parameter values. The first set is a forced response experiment, with a sinusoidal input over a $40-90 \mathrm{~Hz}$ frequency sweep. The signal is controlled using a custom LabVIEW program to maintain a constant acceleration of $2.27 \pm 0.1 \mathrm{~m} / \mathrm{s}^{2}$, so as to decouple the test stand dynamics from the device dynamics. The voltage output across varying load resistances is then collected into the LabVIEW program at a sampling rate of $10 \mathrm{kHz}$ for a duration of one second at each distinct frequency value. The peak-to-peak voltage of the largest signal within that 1 -second time interval is logged for each corresponding frequency value. From these tests, the natural frequency, Young's modulus, voltage, and power output for our selected constant acceleration source are experimentally found.

In the second set of tests the free response of our device is analyzed by using another custom LabVIEW program to input an impulse signal with an arbitrary amplitude. After the impulse signal is generated, the output voltage is measured at a sampling rate of $20 \mathrm{kHz}$, and the decay rate is analyzed. From these tests, previous parameter values are able to be verified as well as estimate other values such as the damping constants and electromechanical coupling constant.

\section{Results}

Using the setup described in the previous section, the design was targeted to have a resonant frequency at $60 \mathrm{~Hz}$, the excitation frequency for VEH's attached to devices powered by the primary outlet line frequency in the US. With the shaker's excitation level set to a constant $2.27 \pm 0.1 \mathrm{~m} / \mathrm{s}^{2}$, a frequency sweep from $40 \mathrm{~Hz}$ to $90 \mathrm{~Hz}$ was performed to determine the resonant frequency of the $\mathrm{VEH}$ device, as well as yield the voltage and power performance when analyzed using varying resistive loads, see Figures 5 and 6 . From the plots, it is observed that the resonant frequency, the location of peak voltage and power, is at $61 \mathrm{~Hz}$. This is within $2 \%$ of the targeted value and corresponds to an estimated structural spring constant of $181.6 \mathrm{~N} / \mathrm{m}$. Young's modulus was experimentally determined to be $530 \mathrm{MPa}$ by applying (8), using each of the measured values mentioned in Section 2, and assuming a Poisson's ratio of 0.4. Likewise, from the plots, a maximum voltage output of $143.6 \mathrm{mV}$ was observed when measuring across a $0.995 \mathrm{M} \Omega$ resistive load and a maximum power output of $2.114 \mu \mathrm{W}$ when measuring across a $2.381 \mathrm{k} \Omega$ resistive load. Normalizing the results with respect to the G-level of the excitation's acceleration, the maximum voltage output is $620.6 \mathrm{mV} / \mathrm{G}$, and the maximum power output is $9.136 \mu \mathrm{W} / \mathrm{G}$.

To verify the results of the frequency sweeps, two impulse tests were performed to confirm the natural frequency and structural spring constant, as well as determine the damping constants. The impulse tests used two different resistive loads, $0.995 \mathrm{M} \Omega$ and $2.381 \mathrm{k} \Omega$, and the free responses were plotted in Figure 7. From the plots, the natural frequency of the free oscillations was observed to be consistent at $61 \mathrm{~Hz}$, which means that the structural spring constant was also consistent between the two tests at $181.6 \mathrm{~N} / \mathrm{m}$. This matches precisely with the forced response values from the frequency sweep tests.

For the $0.995 \mathrm{M} \Omega$ load, the electrical damping was assumed to be near zero, since the Lorentz force is zero at zero current. Therefore with this test, the parasitic damping constant $c_{p}$ is determined directly from the decay rate of the free oscillations, using the approximated values for the spring constant and mass. From the test, the value for $c_{p}$ is approximated to be $0.0671 \mathrm{~kg} / \mathrm{s}$.

Using the same technique of measuring the decay rate of the oscillations, the damping constant for the $2.381 \mathrm{k} \Omega$ load 


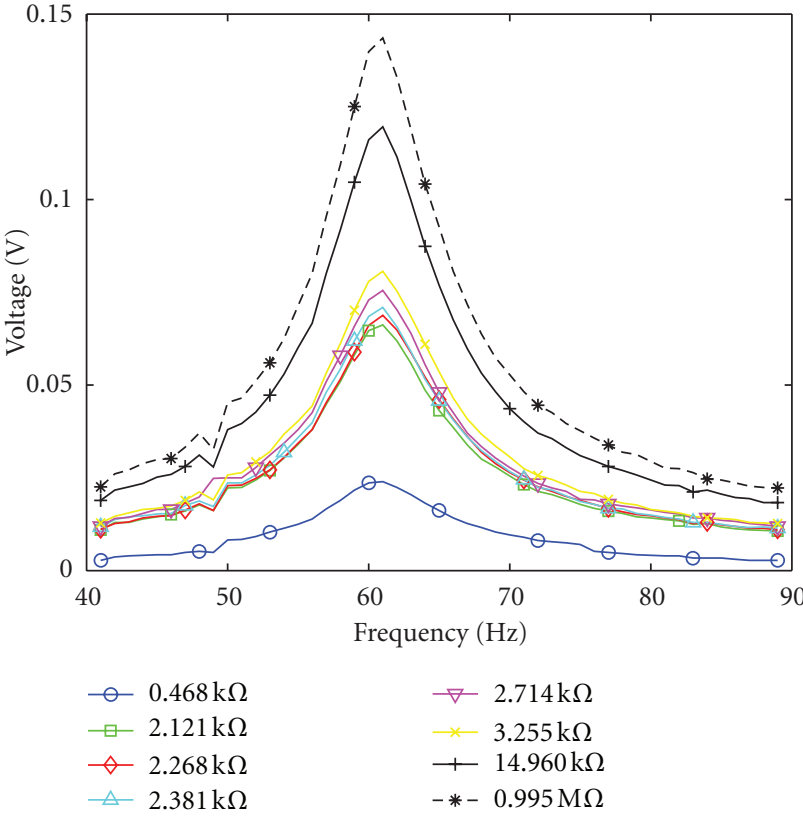

FIGURE 5: Voltage output as function of frequency and resistive load.

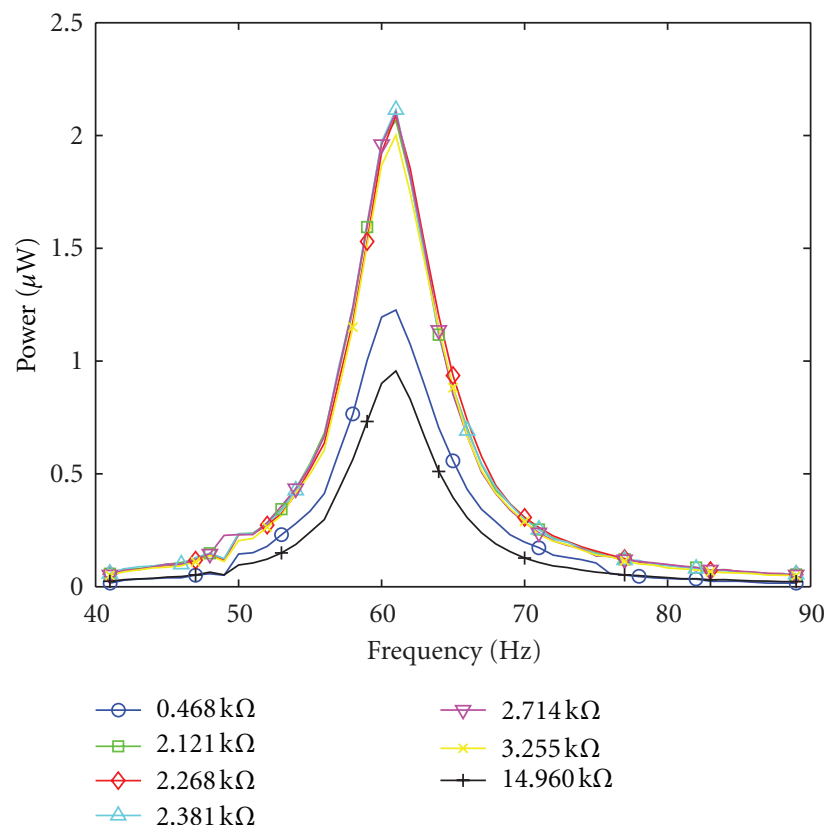

Figure 6: Power output as function of frequency and resistive load using Ohm's law.

is analyzed. However, with this test the electrical damping is assumed measurable due to the presence of the Lorentz force, so the damping constant is actually a superposition of the parasitic and electrical damping constants. This combined damping constant value was found to be $0.081 \mathrm{~kg} / \mathrm{s}$, which when subtracting the parasitic damping constant yields an electrical damping $c_{e}$ estimated at $0.0139 \mathrm{~kg} / \mathrm{s}$. It is noted that by identifying the mechanical and electrical damping constants, an evaluation of the performance of the VEH can
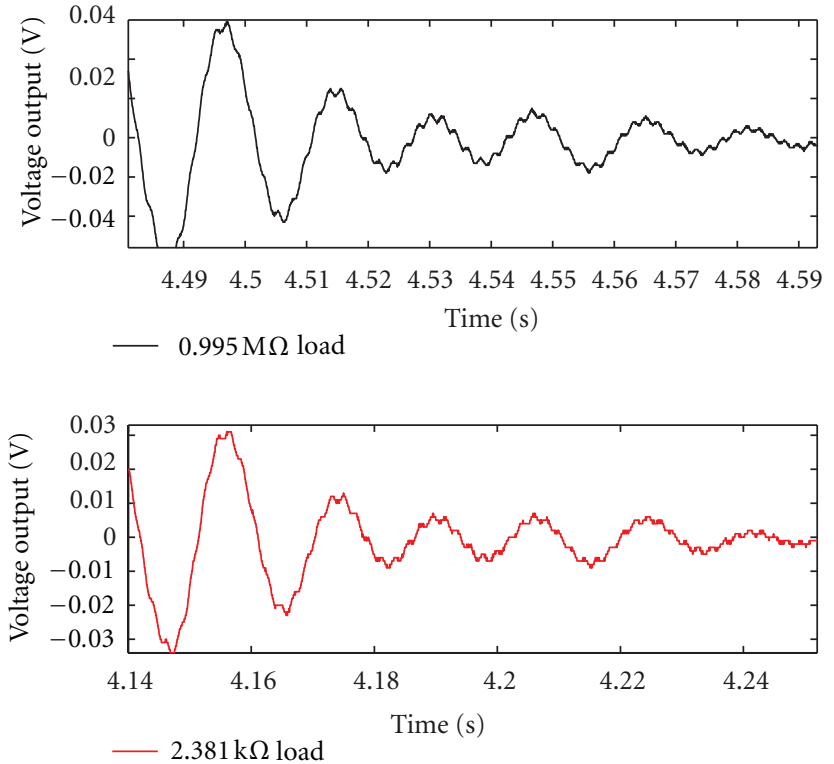

Figure 7: Impulse tests using a $0.995 \mathrm{M} \Omega$ and a $2.381 \mathrm{k} \Omega$ resistive load.

TABLE 3: Comparison of theory with experimental results.

\begin{tabular}{lcc}
\hline $\begin{array}{l}\text { Given parameters: } \\
m=1.236 \mathrm{~g}, n=2,\end{array}$ & Theory & Experiment \\
$\nu=0.4$ & & \\
\hline Natural frequency & $60[\mathrm{~Hz}]$ & $61[\mathrm{~Hz}]$ \\
$k_{\text {helix }}$ & $43.9[\mathrm{~N} / \mathrm{m}]$ & $45.4[\mathrm{~N} / \mathrm{m}]$ \\
$b$ & $400[\mu \mathrm{m}]$ & $372[\mu \mathrm{m}]$ \\
$t$ & $160[\mu \mathrm{m}]$ & $163.5[\mu \mathrm{m}]$ \\
$D$ & $1.04[\mathrm{~mm}]$ & $1.01[\mathrm{~mm}]$ \\
$K_{1}$ & 1.94 & 1.99 \\
$K_{2}$ & 0.317 & 0.304 \\
$K_{w}$ & 1.23 & 1.24 \\
$E$ & $533[\mathrm{MPa}]$ & $530[\mathrm{MPa}]$ \\
$m_{\max } @ 2.27\left[\mathrm{~m} / \mathrm{s}^{2}\right]$ & $73[\mathrm{~g}]^{*}$ & $70[\mathrm{~g}]^{*}$ \\
\hline${ }^{*}$ Assuming a $\sigma_{\max }=25 \mathrm{MPa}[14]$. &
\end{tabular}

be made. According to (6) when the parasitic and electrical damping values are equal, maximum power is generated. Thus, the current design is not optimized, and the electromechanical performance of this device can be further improved. One way to increase the electrical damping, or electromechanical coupling, is to bring the magnetic coil closer to the oscillating magnet structure, increasing the number of turns within the magnetic flux gradient. From this change, the electromechanical coupling $\vec{K}$, estimated at a value of $7.903 \mathrm{~Wb} / \mathrm{m}$ using (7), can be increased. Table 3 is included to summarize all the findings. 
TABLE 4: The literature analysis.

\begin{tabular}{|c|c|c|c|c|c|c|}
\hline Generator & Freq $(\mathrm{Hz})$ & Material & $\begin{array}{l}\text { Young's } \\
\text { modulus } \\
(\mathrm{GPa})\end{array}$ & $\begin{array}{c}\text { Largest } \\
\text { feature size } \\
(\mathrm{mm})\end{array}$ & $\begin{array}{l}\text { Density } \\
\mathrm{g} / \mathrm{cm}^{3}\end{array}$ & $\begin{array}{l}\text { Spring dimensions } \\
\qquad(\mathrm{mm})\end{array}$ \\
\hline This work EM & 61 & HDDA & 0.53 & 3.4 & 1.011 & $\begin{array}{c}0.160 \times 0.400 \text { springs }, \\
3.4 \text { height }\end{array}$ \\
\hline Beeby, 2007 [24] EM & 52 & $\begin{array}{l}\text { Double-polished single } \\
\text { crystal silicon wafers }\end{array}$ & 185 & 9 & 2.329 & $9 \times 3 \times 0.050$ beam \\
\hline Ching, 2001 [25] EM & 110 & Copper & $110-128$ & 4 & 8.94 & 4 radius planar spring \\
\hline White, 2001 [26] PZ & 80 & AISI 316 steel and PZT & $\begin{array}{l}193 \text { and } \\
49-63\end{array}$ & 23 & $\begin{array}{c}8 \text { and } \\
7.5-7.6\end{array}$ & $\begin{array}{c}\text { Trapezoid beam, } 23 \\
\text { base, } 11 \text { top, and } 10 \\
\text { height }\end{array}$ \\
\hline Park, 2010/11 [27, 28] EM & 54 & 100 silicon wafer & 185 & 8 & 2.329 & $\begin{array}{c}8 \times 8 \times 0.04 \text { planar } \\
\text { spring }\end{array}$ \\
\hline Serre, 2007 [29] EM & 920 & $\begin{array}{l}\text { Kapton polymer } \\
\text { membrane }\end{array}$ & 2.5 & 11 & $\begin{array}{c}2.7552 \\
\text { slugs/ft3 }\end{array}$ & $11 \times 11 \times 0.128$ \\
\hline Yang, 2009 [30] EM & $350-600$ & Acrylic & 3.2 & 54 & 1.4 & $54 \times 6 \times 1$ beam \\
\hline Swallow, 2008 [9] PZ & $25-100$ & Copper and PFC & $\begin{array}{c}110-128 \\
\text { and } 50[31]\end{array}$ & 171 & $\begin{array}{l}8.94 \text { and } \\
7.65\end{array}$ & $171 \times 22$ \\
\hline
\end{tabular}

\section{Conclusion}

To conclude, a comparison of this spring design with other small-scale VEH structures in the literature is given, see Table 4. Of notable distinction, it is observed that since the $\mathrm{P} \mu \mathrm{SL}$ process utilizes polymeric material, this work has the lowest Young's modulus (530 MPa) and density $\left(1.011 \mathrm{~g} / \mathrm{cm}^{3}\right)$ for a VEH spring structure to date. The spring structure also has the smallest "largest feature size" for a resonating structure in the sub- $100 \mathrm{~Hz}$ range due to its $3 \mathrm{D}$ geometric design. Where the largest feature size is defined as the largest feature length (length, width, or thickness) of the resonating structure. Thus, the claim is that the $\mathrm{P} \mu \mathrm{SL}$ fabrication method, along with the 3D helical design choice, yields the most compact VEH spring structure to date that is tuned to resonate at sub- $100 \mathrm{~Hz}$. To justify this claim, the spring structure in this work is compared to the smallest "largest feature size" of a sub- $100 \mathrm{~Hz}$ resonance $\mathrm{VEH}$, which is described in Beeby et al. at 2.4 times this work's value, or $8 \mathrm{~mm}$ [24]. Additionally, when slightly relaxing the sub$100 \mathrm{~Hz}$ resonance constraint and simply comparing with the smallest "largest feature size," the work described in Ching et al. is still larger than this work's value with a $4 \mathrm{~mm}$ radius planar spring and with a resonant frequency that is 1.8 times higher at $110 \mathrm{~Hz}$ [25].

Thus, the key attributes of this paper are the widening of the design space and the tuning of the Young's modulus via the P $\mu$ SL fabrication process and the UV postcure time. Using the helical spring design, these attributes have been demonstrated with the fabrication of a $3 \mathrm{D}$ polymeric structure with feature sizes ranging from $160 \mu \mathrm{m}$ to $3.4 \mathrm{~mm}$ and a Young's modulus of $530 \mathrm{MPa}$. The result is a VEH device resonating at $61 \mathrm{~Hz}$ with a 1.236 gram magnetic load mass. As for the electromagnetic VEH performance, when excited at resonance by a uniaxial vibration source, the open circuit voltage was $620.6 \mathrm{mV} / \mathrm{G}$, and the power output was $9.136 \mu \mathrm{W} / \mathrm{G}$. Although the current system delivers moderate electric power output, it is noted that it was not optimized for maximum power performance but instead for creating a compact spring structure that was able to resonate near $60 \mathrm{~Hz}$. The maximum power performance could be increased beyond these values through optimizing the mechanical and electrical damping, as shown in (6). However, with the reported design and an open circuit quality factor of 6.94 , it can be seen that by trading off lowered peak power performance at resonance, a broader operation frequency range of nearly $10 \mathrm{~Hz}$ is gained, which is useful if the environmental vibration frequency is slightly variable. Future work is aimed at improving the performance of these spring structures, specifically the VEH performance values, by exploring the effect of varying the dimensions and modulus of the microstructures, as well as fabricating coils to optimize the electromechanical coupling. To conclude, the use of $\mathrm{P} \mu \mathrm{SL}$ and the postcuring process has allowed for the creation of truly miniaturized, 3D VEH with low resonant frequencies. With further exploration of this technique, the competitiveness of using $\mathrm{VEH}$ in portable power devices is closer to becoming a reality.

\section{Acknowledgments}

The authors would like to thank Beau Becker for his work in the machine shop fabricating aluminum stands and magnet wire coils and Zhen Zhang for his advice and theories. This work is supported by the National Science Foundation under Grant number CMMI-1130948, CMMI-0955195, and CMMI-0751621 and a booster award from the Initiative for Sustainability and Energy at Northwestern (ISEN). The authors would also like to thank Dr. Jim Beckwith from the Department of Intelligence for supporting this work through the Intelligence Community Postdoctoral Fellowship Program. 


\section{References}

[1] I. Marin et al., "Wireless Sensor Networks: A Survey on Ultra-Low Power-Aware Design," in Proceedings of the World Academy of Science, Engineering and Technology, vol. 8, pp. 4449, 2005.

[2] L. Chao, C. Y. Tsui, and W. H. Ki, "A batteryless vibrationbased energy harvesting system for ultra low power ubiquitous applications," in Proceedings of the IEEE International Symposium on Circuits and Systems (ISCAS '07), vol. 1-11, pp. 13491352, May 2007.

[3] D. Yeager, F. Zhang, A. Zarrasvand, N. T. George, T. Daniel, and B. P. Otis, "A $9 \mu \mathrm{A}$, addressable Gen2 sensor tag for biosignal acquisition," IEEE Journal of Solid-State Circuits, vol. 45, no. 10, pp. 2198-2209, 2010.

[4] B. Yahya and J. Ben-Othman, "Towards a classification of energy aware MAC protocols for wireless sensor networks," Wireless Communications and Mobile Computing, vol. 9, no. 12, pp. 1572-1607, 2009.

[5] D. P. Arnold, "Review of microscale magnetic power generation," IEEE Transactions on Magnetics, vol. 43, no. 11, pp. 3940-3951, 2007.

[6] S. P. Beeby, M. J. Tudor, and N. M. White, "Energy harvesting vibration sources for microsystems applications," Measurement Science and Technology, vol. 17, no. 12, article no. R01, pp. R175-R195, 2006.

[7] M. Umeda, K. Nakamura, and S. Ueha, "Analysis of the transformation of mechanical impact energy to electric energy using piezoelectric vibrator," Japanese Journal of Applied Physics, Part 1: Regular Papers and Short Notes and Review Papers, vol. 35, no. 5 B, pp. 3267-3273, 1996.

[8] S. Wang, K. H. Lam, C. L. Sun et al., "Energy harvesting with piezoelectric drum transducer," Applied Physics Letters, vol. 90, no. 11, Article ID 113506, 3 pages, 2007.

[9] L. M. Swallow, J. K. Luo, E. Siores, I. Patel, and D. Dodds, "A piezoelectric fibre composite based energy harvesting device for potential wearable applications," Smart Materials and Structures, vol. 17, no. 2, Article ID 025017, 2008.

[10] P. Glynne-Jones, M. J. Tudor, S. P. Beeby, and N. M. White, "An electromagnetic, vibration-powered generator for intelligent sensor systems," Sensors and Actuators, A, vol. 110, no. 1-3, pp. 344-349, 2004.

[11] K. Jin, X. Ruan, M. Yang, and M. Xu, "A hybrid fuel cell power system," IEEE Transactions on Industrial Electronics, vol. 56, no. 4, pp. 1212-1222, 2009.

[12] S. Kulkarni, S. Roy, T. O'Donnell, S. Beeby, and J. Tudor, "Vibration based electromagnetic micropower generator on silicon," Journal of Applied Physics, vol. 99, no. 8, Article ID 08P511, 2006.

[13] C. Sun, N. Fang, D. M. Wu, and X. Zhang, "Projection microstereolithography using digital micro-mirror dynamic mask," Sensors and Actuators, A, vol. 121, no. 1, pp. 113-120, 2005.

[14] E. Manias, J. Chen, N. Fang, and X. Zhang, "Polymeric micromechanical components with tunable stiffness," Applied Physics Letters, vol. 79, no. 11, pp. 1700-1702, 2001.

[15] A. Marin, S. Bressers, and S. Priya, "Multiple cell configuration electromagnetic vibration energy harvester," Journal of Physics $D$, vol. 44, no. 29, Article ID 295501, 2011.

[16] F. Zhou, Y. Bao, Cao W. et al., "Hiding a realistic object using a broadband terahertz invisibility cloak," Scientific Reports, vol. 1 , article 78, 2011.

[17] C. Xia and N. X. Fang, "3D microfabricated bioreactor with capillaries," Biomedical Microdevices, vol. 11, no. 6, pp. 1309$1315,2009$.
[18] H. Lee, C. Xia, and N. X. Fang, "First jump of microgel; Actuation speed enhancement by elastic instability," Soft Matter, vol. 6, no. 18, pp. 4342-4345, 2010.

[19] X. N. Jiang, C. Sun, X. Zhang, B. Xu, and Y. H. Ye, "Microstereolithography of lead zirconate titanate thick film on silicon substrate," Sensors and Actuators A, vol. 87, no. 1-2, pp. 72-77, 2000.

[20] K. S. Ho, R. J. Bradley, D. R. Billson, and D. A. Hutchins, "Micro-stereolithography as a transducer design method," Ultrasonics, vol. 48, no. 1, pp. 1-5, 2008.

[21] C. Xia, H. Lee, and N. Fang, "Solvent-driven polymeric micro beam Device," Journal of Micromechanics and Microengineering, vol. 20, no. 8, Article ID 085030, 2010.

[22] C. Cepnik, O. Radler, S. Rosenbaum, T. Ströhla, and U. Wallrabe, "Effective optimization of electromagnetic energy harvesters through direct computation of the electromagnetic coupling," Sensors and Actuators A, vol. 167, no. 2, pp. 416$421,2011$.

[23] S. Roundy, P. K. Wright, and J. Rabaey, "A study of low level vibrations as a power source for wireless sensor nodes," Computer Communications, vol. 26, no. 11, pp. 1131-1144, 2003.

[24] S. P. Beeby, R. N. Torah, M. J. Tudor et al., "A micro electromagnetic generator for vibration energy harvesting," Journal of Micromechanics and Microengineering, vol. 17, no. 7, article no. 007, pp. 1257-1265, 2007.

[25] N. N. H. Ching, H. Y. Wong, W. J. Li, P. H. W. Leong, and Z. Wen, "A laser-micromachined vibrational to electrical power transducer for wireless sensing systems," in Proceedings of the 11th International Conference on Solid-State Sensors and Actuators (Transducers '01 / Eurosensors XV), vol. 1-2, pp. 3841, Munich, Germany, 2001.

[26] P. Glynne-Jones, S. P. Beeby, and N. M. White, "Towards a piezoelectric vibration-powered microgenerator," IEE Proceedings: Science, Measurement and Technology, vol. 148, no. 2, pp. 68-72, 2001.

[27] J. C. Park, D. H. Bang, and J. Y. Park, "Micro-fabricated electromagnetic power generator to scavenge low ambient vibration," IEEE Transactions on Magnetics, vol. 46, no. 6, pp. 1937-1942, 2010.

[28] J. C. Park and J. Y. Park, "A bulk micromachined electromagnetic micro-power generator for anambient vibration-energyharvesting system," Journal of the Korean Physical Society, vol. 58, no. 5, pp. 1468-1473, 2011.

[29] C. Serre, A. Pérez-Rodríguez, N. Fondevilla, J. R. Morante, J. Montserrat, and J. Esteve, "Vibrational energy scavenging with Si technology electromagnetic inertial microgenerators," Microsystem Technologies, vol. 13, no. 11-12, pp. 1655-1661, 2007.

[30] B. Yang, C. Lee, W. Xiang et al., "Electromagnetic energy harvesting from vibrations of multiple frequencies," Journal of Micromechanics and Microengineering, vol. 19, no. 3, Article ID 035001, 2009.

[31] R. A. de Oliveira, P. Neves, J. T. Pereira, and A. P. Pohl, “Analysis of mechanical properties of a photonic crystal fiber bragg grating acousto-optic modulator," in Proceedings of the 1st Workshop on Specialty Optical Fibers and Their Applications, vol. 1055, pp. 117-120, São Pedro, Brazil, 2008. 

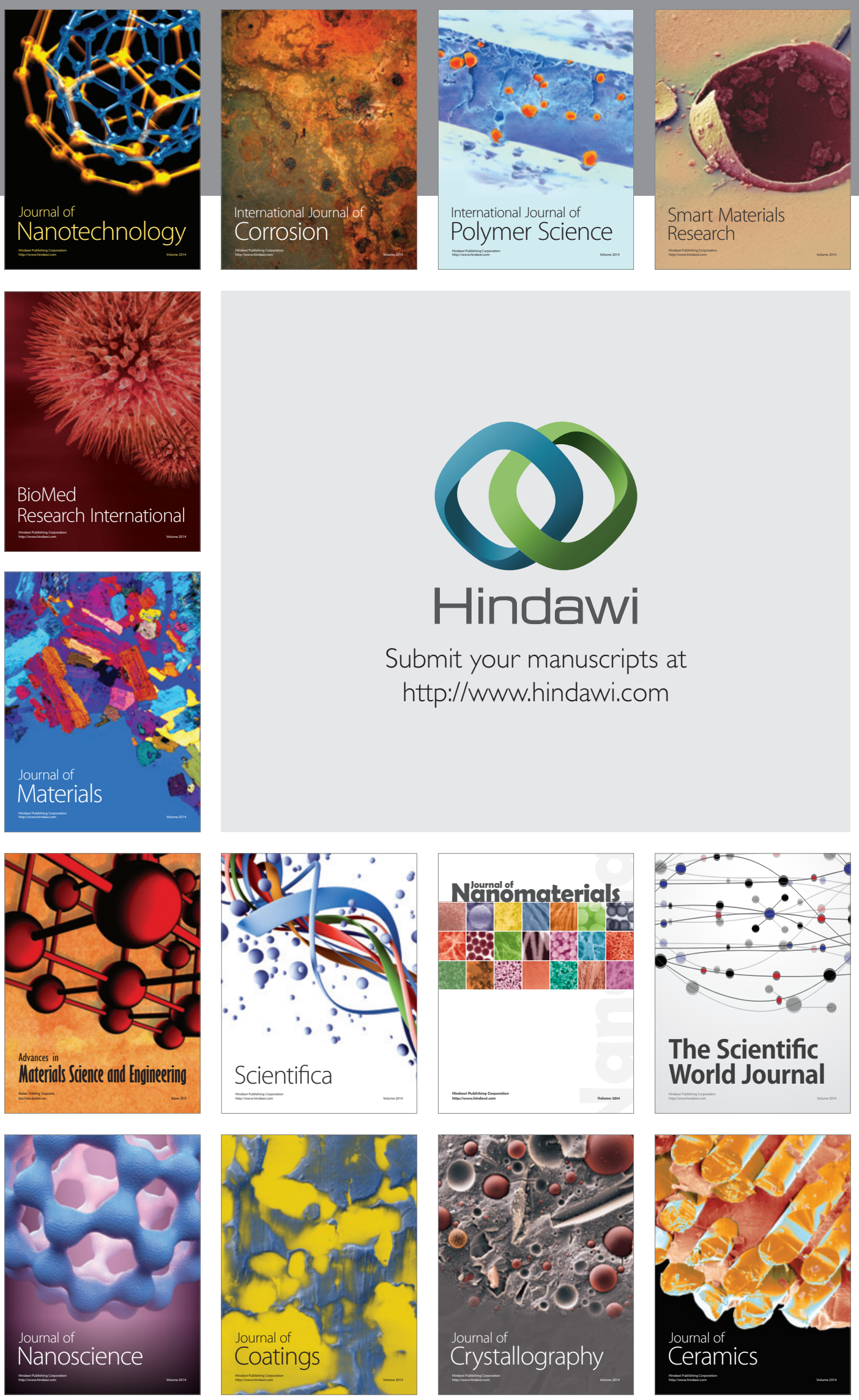

The Scientific World Journal

Submit your manuscripts at

http://www.hindawi.com

\section{World Journal}

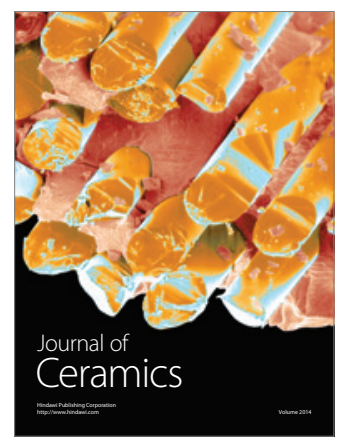

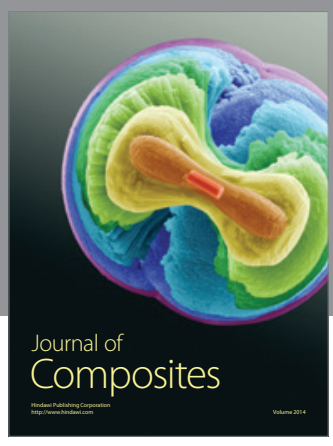
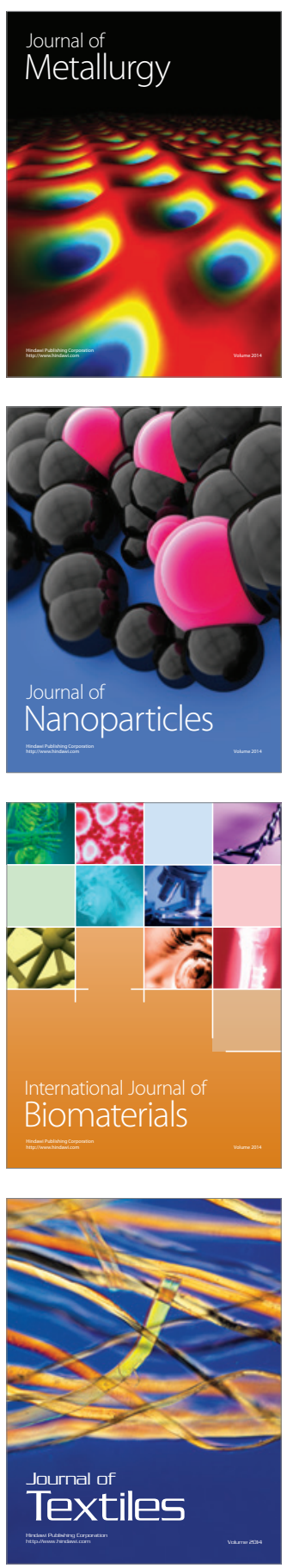